\title{
Whole-mount in situ hybridization in the Rotifer Brachionus plicatilis representing a basal branch of lophotrochozoans
}

\author{
Louis A. Boell • Gregor Bucher
}

Received: 21 December 2007 / Accepted: 10 June 2008 / Published online: 2 July 2008

(C) The Author(s) 2008

\begin{abstract}
In order to broaden the comparative scope of evolutionary developmental biology and to refine our picture of animal macroevolution, it is necessary to establish new model organisms, especially from previously underrepresented groups, like the Lophotrochozoa. We have established the culture and protocols for molecular developmental biology in the rotifer species Brachionus plicatilis Müller (Rotifera, Monogononta). Rotifers are nonsegmented animals with enigmatic basal position within the lophotrochozoans and marked by several evolutionary novelties like the wheel organ (corona), the median eye, and the nonpaired posterior foot. The expression of $\mathrm{Bp}$ Pax-6 is shown using whole-mount in situ hybridization. The inexpensive easy culture and experimental tractability of Brachionus as well as the range of interesting questions to which it holds the key make it a promising addition to the "zoo" of evo-devo model organisms.
\end{abstract}

Keywords Lophotrochozoa $\cdot$ Rotifer $\cdot$ Cleavage $\cdot$ Pax6

Communicated by D. A. Weisblat

Electronic supplementary material The online version of this article (doi:10.1007/s00427-008-0234-z) contains supplementary material, which is available to authorized users.

L. A. Boell

Max-Planck-Institut für Evolutionsbiologie,

August-Thienemann-Straße 2,

24306 Plön, Germany

G. Bucher $(\square)$

Abt. für Entwicklungsbiologie,

Johann Friedrich-Blumenbach-Institut,

Georg-August-Universität,

Justus-von-Liebig-Weg 11,

37077 Göttingen, Germany

e-mail: gbucher1@uni-goettingen.de

\section{Introduction}

One aim of evo-devo research is the reconstruction of ancestral embryonic patterning principles that were present in the urbilaterian, i.e., the last common ancestor of bilaterian animals. To this end, expression patterns of orthologous genes are compared across the animal kingdom. Similarities across phyla indicate that the respective genes might have had similar functions already in the Urbilateria. The other major goal is to understand the evolution of morphological novelties. Here, the patterning principle identified for a taxon-specific structure is compared to the likely ancestral state which leads to hypotheses about the changes that were required in the regulatory gene network for the evolution of this trait.

Unfortunately, most protostome model systems belong to the Ecdysozoans while other groups remain notoriously underrepresented. Another model system bias is the overrepresentation of segmented over nonsegmented taxa.

The cryptic species complex of Brachionus plicatilis Müller (Rotifera) comprises at least 14 species (Suatoni et al. 2006) which are small cyclically parthenogenetic euryhaline marine invertebrates. They display a nonspiralian cleavage and direct development and are not segmented. Several unique structures distinguish rotifers including the anterior wheel organ (corona), a complex feeding organ (mastax), and a single posterior "foot". Another singularity of many rotifer species is the single cerebral eye (see Fig. 1). Their phylogenetic position remains unclear (Welch 2001). Recently, it has been proposed that rotifers are part of a basal lophotrochozoan group (Dunn et al. 2008) while also a sister group relationship to lophotrochozoans has been proposed earlier as part of a taxon called Platyzoa. Some work on rotifer development has been done (Boschetti et al. 2005; Castellano Paez et al. 1988; 


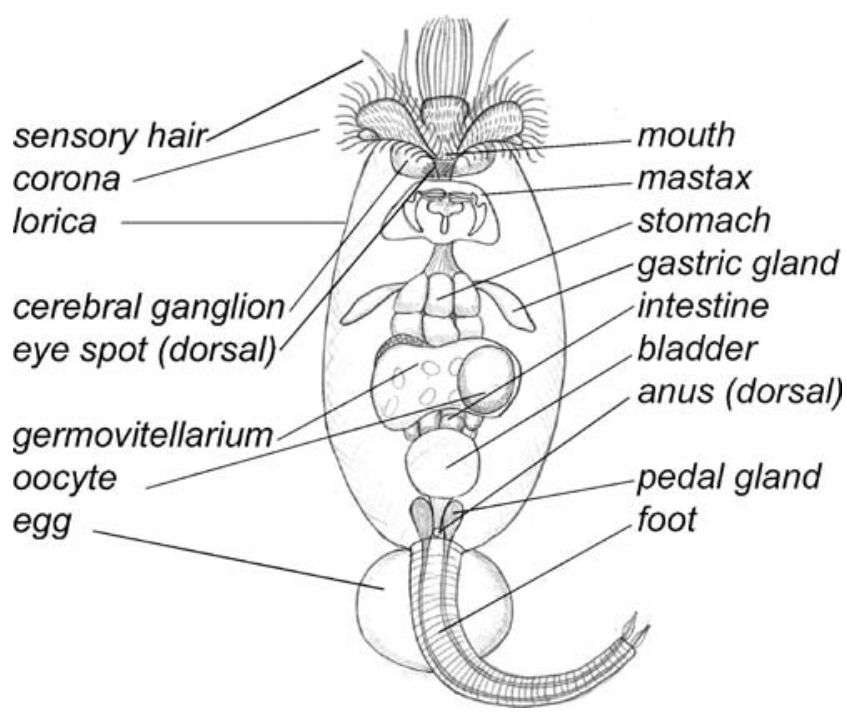

Fig. 1 Schematic of $B$. plicatilis. Shown is an adult female in a ventral view carrying one shed egg. The single eyespot is located on the dorsal side of the cerebral ganglion. The mastax is a complex apparatus required to grind food. The corona (wheel organ) is a highly ciliated structure used for locomotion and feeding. Muscles, excretory cells, and nerves have been omitted for simplicity. Redrawn from Storch and Welsch 1999

deBeauchamp 1956; Lechner 1966; Pray 1965; Nachtwey 1925; Tannreuther 1920; Tessin 1886; Zelinka 1891; Jennings 1896) but gene expression data are lacking. This neglect may be due to the methodological difficulties presented by the small size of rotifers and their very resistant eggshell. We have established B. plicatilis as a rotifer model system amenable to whole-mount in situ staining and confocal imaging using fluorescent dyes.

\section{Material and methods}

Rotifer culture and egg collection

B. plicatilis were purchased from the company Interaquaristics (Biedenkopf, Germany). Sequencing of the cytochrome c oxidase subunit I (COI; as described in Gomez et al. 2002) and comparison with the COI sequences used in the most recent phylogeny given in Suatoni et al. (2006) revealed highest similarity to the Nevada clade with $98 \%$ identity to the Notojiima isolate and $90 \%$ identity to the "Little Fish Lake, Nevada" isolate. They were reared in marine water prepared from a commercial salt mixture ("Hobby marin Meersalz") by solving $30 \mathrm{~g}$ of the mixture per liter of tap water. The cultures were kept in 1-1 bottles or Erlenmeyer flasks and fed on separately cultured marine phytoplankton (Interaquaristics) that was grown in seawater as well. The latter material was kept in an 1-1 bottle, aerated with an aquarium pump (Elite $799,2 \mathrm{~W}, 500 \mathrm{~cm}^{3} / \mathrm{min}$ ), provided with light by a full spectrum fluorescent lamp (SUN-GLO, $15 \mathrm{~W}$ ), and fertilized by adding a teaspoon per week of "Substral" universal fertilizer (Scotts Celaflor, Ingelheim, Germany). Rotifers were fed by replacing their medium with phytoplankton medium. To avoid contamination of RNA or DNA preparations with nonsequenced organisms, rotifers can be fed on yeast (dissolving $10 \mathrm{~g}$ of dried baker's yeast in 50-ml tap water and feeding $1.5 \mathrm{ml}$ of this solution per liter rotifer culture every 2 days). At $28^{\circ} \mathrm{C}$ and with sufficient food supply, Brachionus reproduces parthenogenetically, roughly doubling population size in a day. From time to time, the cultures were checked for the presence of unwanted organisms (e.g., Nematodes, Gnathostomulids, or Protozoans). Note that at high population densities the reproduction will switch from parthenogenetic to sexual. However, we have never observed male individuals in our stock, indicating that we predominantly worked under parthenogenetic conditions.

\section{Collection and fixation of embryos}

The rotifers and eggs were collected from the medium by filtration through a cell culture sieve (Corning, Bad Hoevedorp, Netherlands) with a pore width of $74 \mu \mathrm{m}$ which was fixed inside a plastic funnel. They were then transferred into plastic vials (50 $\mathrm{ml}$ falcons) with a Pasteur pipette. In order to separate the embryos from their mother animals, the filled 50-ml plastic vials were hit against the edge of the bench for five to ten times as powerfully as possible, just avoiding breaking the vials. The embryos settle to the bottom of the vial, while the adults resume swimming and can be removed with the supernatant. The embryos continue development normally after this procedure.

\section{Fixation and storage}

Embryos are fixed in formaldehyde (FA) solution in $1.5-\mathrm{ml}$ Eppendorf cups. Settling of the embryos takes about $2 \mathrm{~min}$ (note: embryos are damaged upon centrifugation). In order to avoid implosion upon sudden changes of osmolarity, we fixed embryos by raising the FA concentration by $0.25 \%$ in 12 steps of 1-min duration each. Embryos were left in the final 3\% FA solution for further 8 min and then washed in phosphate buffer with Tween (PBT). The supernatant was removed and replaced with PBT. This procedure, referred to as washing (W, W20 meaning 20-min incubation after addition of the buffer) in the following, was performed three times. In order to avoid damage through sudden change of osmolarity, transfer into methanol requires three subsequent $\mathrm{W}$ steps with a mixture of PBT-methanol in a ratio of 5:1, 3:1, and 1:1. Finally, embryos are washed three times in methanol and stored at $-20^{\circ} \mathrm{C}$. For experiments, 
the embryos are brought back to PBT by the reverse procedure.

Permeabilization of the eggshell

The shell of the rotifer eggs is impermeable to most molecules. Because of the small size of the eggs ( 125-•mlong diameter) and elasticity of the eggshell, manual preparation was not possible. Attempts to degrade the shell using chemicals $(2 \mathrm{M} \mathrm{NaOH}, 37 \% \mathrm{HCl}, \mathrm{NH} 4 \mathrm{OH}$, lactic acid, perchloric acid, propionic acid, formic acid, phosphoric acid, sulfuric acid, and bleach (sodium hypochlorite), adding up to $30 \%$ volume of the culture medium) or enzymes (proteinase $\mathrm{K}$, pronase) also in combination with thioglycolic acid, cysteine, and dithiothreitol in different combinations, concentrations, and incubation times proved either ineffective or destroyed the embryos while leaving the eggshells intact. A mechanical treatment was successful: We glued fine-grained sandpaper (European Federation of European Producers of Abrasives-P standard: K600 (size of grain $25.8 \mu \mathrm{m}$ ) which corresponds to an intermediate between the American National Standards Institute standards 360 and 400 and the Japanese Industrial Standards $\mathrm{J} 600$ and J700) to a "pestle" made of a syringe plunger.
Embryos were transferred to a glass staining block with concave bottom in the smallest possible volume of PBT. Using the plunger, the embryos were "ground" repeatedly against the concave ground of a staining block as a mortar. This ruptures the eggshell but also damages embryos. Therefore, the effect must be controlled under the binocular to optimize the portion of damaged eggshells vs. destroyed embryos. Within a batch, about $10 \%$ of embryos of all stages can be permeabilized.

\section{Autofluorescence and staining}

Rotifer embryos exhibit strong autofluorescence at a variety of wavelengths (see for instance 4',6-diamidino2-phenylindole (DAPI) staining in Fig. 2). We tested the following filter sets (in order of increasing excitation wavelength): Zeiss filter sets 01 (BP365/12), 06 (BP 436/10), 09 (BP 450-490), 15 and 20 (both BP 546/12), 38 (BP 470/ 20 ), and 46 (BP500/20). Only with the filter set 06 was the autofluorescence of rotifer embryos negligible. Neither storage in methanol nor diet (phytoplankton vs. yeast) affected the autofluorescence. After a stay in methanol, embryos are accessible to DAPI (molecular probes, Invitrogen) and other small fluorescent dyes.
Fig. 2 Development of $B$. plicatilis as visualized by F-actin staining. The animal (prospective posterior) pole is oriented upward in all panels. Cleavage is holoblastic. a The first cleavage is unequal and produces a small cell at the animal pole and a large cell at the vegetal pole. b-f The cell at the animal pole and its derivatives subsequently cleave more or less equally. f-i They internalize the vegetal cell and its derivatives by epiboly. The vegetal cell continues to cleave unequally. $\mathbf{j}-\mathbf{l}$ organogenesis and differentiation

A
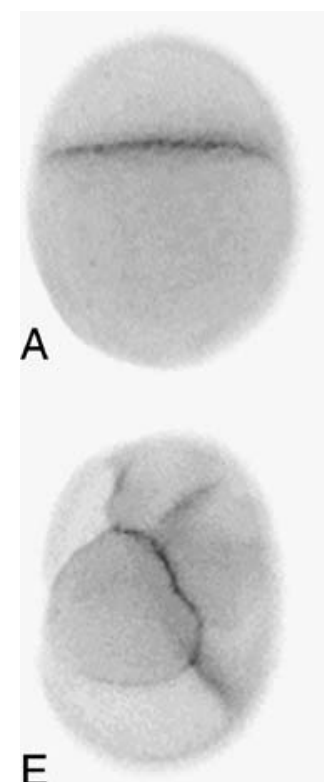

E

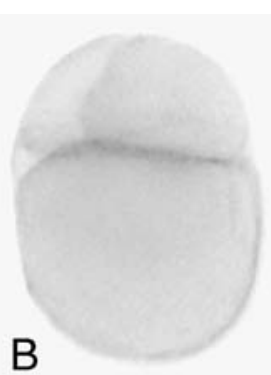

B
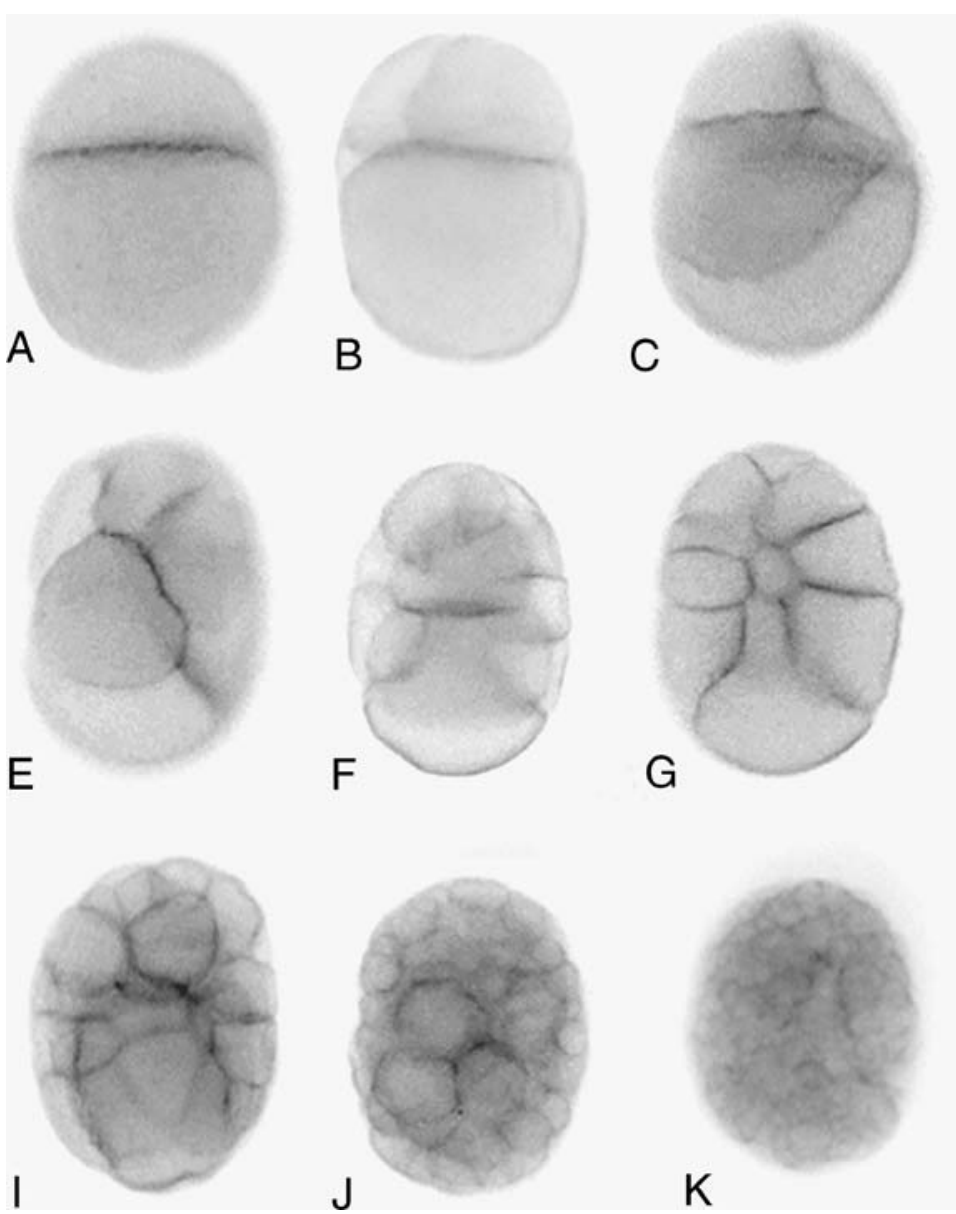

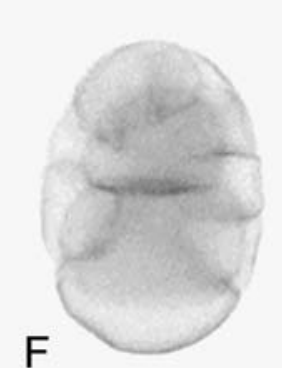

F

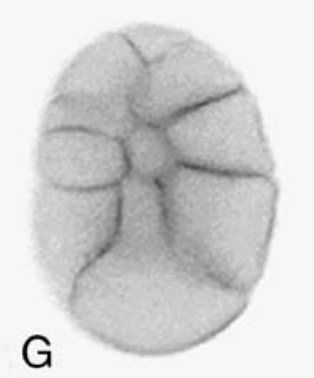

G

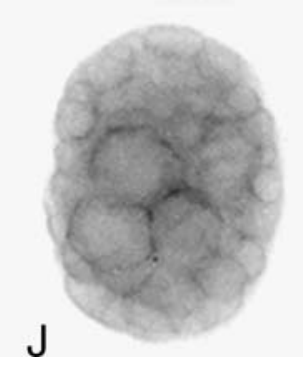

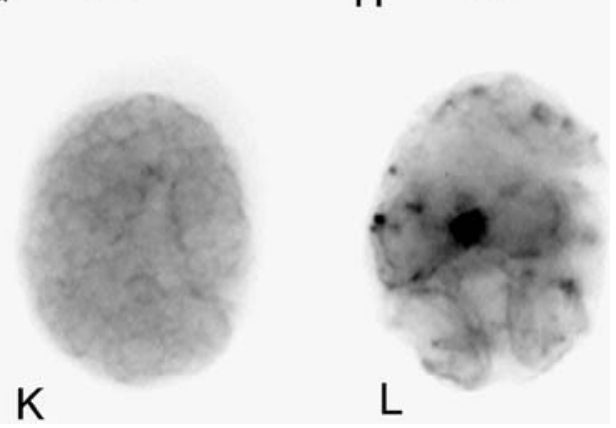

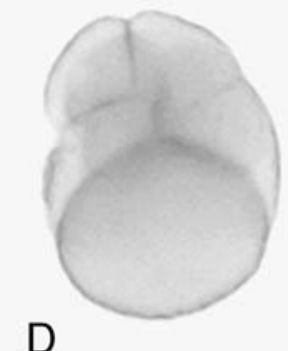

D

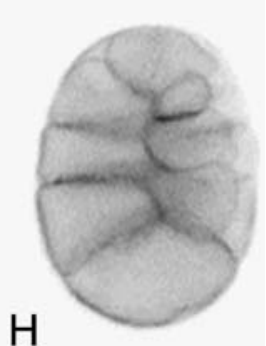

$\mathrm{H}$ 
Actin staining of the cytoskeleton

After permeabilization of the eggshell, embryos are accessible to Phalloidin/Alexa 488 (Invitrogen), allowing visualization of the actin cytoskeleton that marks the outlines of the blastomeres. As proteins may not be denatured for actin staining, PBT has to be replaced with phosphate-buffered saline (PBS) as a washing buffer during fixation and preparation, and embryos may not be washed or stored in methanol. After fixation and permeabilization of the eggshell, embryos were permeabilized with $5 \mu \mathrm{l}$ $10 \mathrm{mg} / \mathrm{ml}$ proteinase $\mathrm{K}$ per milliliter PBS for $2 \mathrm{~min}$ at $65^{\circ} \mathrm{C}$ in a shaker. The proteinase was washed out by W3 with PBS. RNA was degraded by a treatment with $10 \mu \mathrm{l}$ RNAseA per milliliter PBS at $37^{\circ} \mathrm{C}$. After $2 \times \mathrm{W}$ with PBS, embryos were stained for $1 \mathrm{~h}$ with $1 \mu$ l Phalloidin/ Alexa 488 in a small volume of PBS and analyzed after $2 \times$ W with $1 \mathrm{ml}$ PBS.

\section{Cloning of Pax-6 and sequence analysis}

Animals were collected and shock-frozen and total RNA was prepared using the Trifast RNA extraction Kit (Peqlab). cDNA was prepared from total RNA using the SMART cDNA synthesis kit (BD Life sciences). Fragments of $B p$ Pax-6 were amplified by polymerase chain reaction (PCR) with nested degenerate primers on cDNA (Wim Damen, pers. communication). The forward ( $\mathrm{fw}$ ) primers lie in the paired domain, the reverse (rev) primers in the homeodomain: Pax-6 fw1: HSGVNQL (5'CA(CT) (AT)(GC) (AGCT) GG(AGCT) GT(AGCT) AA(CT) CA(AG) (CT)T (AGCT) GG(AGCT) GG 3'); Pax-6 fw2: TRQKIVELA (5' AC(AGCT) (AC)G(AGCT) CA(AG) AA(AG) AT(ACT) GT(AGCT) GA(AG) (CT)T(AGCT) GC 3'); Pax-6 rev1: PEARIQVWF (5'AAC CA(AGCT) AC(CT) TG(AGT) AT (AGCT) C(GT)(AGCT) GC(CT) TC(AGCT) GG 3'); Pax6 rev2: EKEFERTHY (5'TA(AG) TG(AGCT) GT(AGCT) $\left.\mathrm{C}(\mathrm{GT})(\mathrm{CT}) \mathrm{TC}(\mathrm{AG}) \mathrm{AA}(\mathrm{CT}) \mathrm{TC}(\mathrm{CT}) \mathrm{TT}(\mathrm{CT}) \mathrm{TC} 3^{\prime}\right)$. The PCRs were performed in $10-\mu l$ volume using the Fermentas PCR kit with a primer concentration of $20 \mu \mathrm{M} / \mu \mathrm{l}$. Template: $1-\mu \mathrm{l}$ cDNA preparation, $\mathrm{H}_{2} \mathrm{O} 5.15 \mu \mathrm{l}, 10 \times$ buffer: $1 \mu \mathrm{l} ; \mathrm{MgCl}_{2}$ solution $0.6 \mu \mathrm{l}$; deoxynucleotide triphosphates $1 \mu \mathrm{l}$; primer fo $0.5 \mu \mathrm{l}$; primer revc $0.5 \mu \mathrm{l}$; Taq $1 \mu \mathrm{l}$. The PCRs were performed in a gradient with annealing temperatures ranging from $40^{\circ} \mathrm{C}$ to $55^{\circ} \mathrm{C}$. Cycling parameters: initial denaturation: $2 \mathrm{~min}$ at $95^{\circ} \mathrm{C}$; denaturation: $30 \mathrm{~s}$ at $95^{\circ} \mathrm{C}$; annealing: $1 \mathrm{~min}$; elongation: $1 \mathrm{~min}$ at $72^{\circ} \mathrm{C}$; number of cycles 25 ; final elongation: $5 \mathrm{~min}$ at $72^{\circ} \mathrm{C}$. PCR products were analyzed on a $1 \%$ agarose gel. Bands of the expected length $(1.2-1.8 \mathrm{~kb})$ were extracted using the Quiaex II gel extraction kit (Quiagen) and the fragments cloned using the TA cloning kit (Invitrogen). Nested rapid amplification of cDNA ends (RACE) PCR was performed: Pax-6/1 (3'): 5'GAA GAG ACC CAG GCC AGG CTG AGA CTG AAG 3'; Pax-6/2 (3' nested): $5^{\prime}$ TCG AGC GCA CAC ACT ACC CGG ACG TGT ATG 3'; Pax-6/3 (5'): 5'GTC GAT CTC GCT GTT CAA ATT GGA GCT GTC 3'; Pax-6/4 (5' nested): 5'GCA TTC GGC AAG TTC ACT GTT GTT CAT GTG 3'. Templates for RACE PCR were prepared using the SMART RACE PCR kit (BD Life sciences), and RACE PCRs were performed with the BD Advantage 2 PCR kit. RACE products were cloned using the TA cloning kit (Invitrogen) and sequenced from both sides using T7 and SP6 primers. One of the positive clones of the Pax-6 5'region was used as a template for the preparation of a digoxigenin (DIG)-labeled antisense RNA probe using the (Roche) DIG labeling kit. The sequences for the alignment were retrieved from the SRS protein database http://srs.ebi.ac.uk/ and their paired and homeodomain boxes aligned using ClustalW with subsequent manual curation. Only clearly aligned positions were used for the phylogenetic analysis. A phylogenetic tree was calculated using the neighbor-joining method as implemented in the MEGA software (version 4; Tamura et al. 2007; http://www.megasoftware.net/). Bootstrap values were calculated based on 1,500 replications.

Whole-mount in situ hybridization

For efficient buffer change, embryos were transferred to small Plexiglas cylinders of 9-mm diameter, with a $54-\mu \mathrm{m}$ pore width gaze mesh at the bottom (Bückmann, Mönchengladbach). These cylinders fitted the wells of a custom-made acrylic well block. The cylinders containing the embryos were moved from one well to the next containing the fresh buffer. Buffer change will be referred to in the following as rinsing (R; R15 means 15-min incubation while gently stirring the well block on a shaking platform). Embryos are brought from methanol to PBT as described above and rinsed two more times in PBT. Postfixation was done by R15 in $1 \mathrm{ml}$ PBT plus $140 \mu \mathrm{l} 37 \%$ formaldehyde. After $5 \times \mathrm{R}$ with PBT, embryos were transferred into an Eppendorf cup with $1 \mathrm{ml}$ PBT. Five microliter proteinase $\mathrm{K}(10 \mathrm{mg} / \mathrm{ml})$ were added and embryos were permeabilized at $65^{\circ} \mathrm{C}$ for 2 min, shaking strongly. The embryos were then brought back into a cylinder and $2 \times \mathrm{R}$ with PBT. Postfixation was done by R15 in $1 \mathrm{ml}$ PBT plus $140 \mu \mathrm{l} 37 \%$ formaldehyde. After $4 \times \mathrm{R}$ with PBT, they were brought into hybridization buffer (Hyb; see below): $\mathrm{R}$ with HybB-PBT 1:1, R with HybB, R with HybA. Embryos were preincubated in $\mathrm{HybA}$ for $1 \mathrm{~h}$ at $65^{\circ} \mathrm{C}$. They were then transferred into an Eppendorf cup and allowed to settle for $5 \mathrm{~min}$ and the supernatant was reduced to approximately $30-\mu l$ volume. A $0.1 \mu 1$ of $1: 10$ diluted DIG-labeled antisense RNA probe was added. Hybridiza- 
tion was performed overnight at $65^{\circ} \mathrm{C}$. Embryos were brought back into PBT by adding $1 \mathrm{ml}$ prewarmed HybB at $65^{\circ} \mathrm{C}$ and subsequent transfer into a Plexiglas cylinder in the well block. Subsequently, R5 in $\mathrm{HybB}$ at $65^{\circ} \mathrm{C}$ (without stirring), $\mathrm{R}$ with $\mathrm{HybB-PBT} 1: 1$ at room temperature, R R R15 with PBT. The embryos were then blocked by R20 with BBT (see below) and antiDIG antibodies (Roche) diluted 1:2,000 were added for $1 \mathrm{~h}$ in BBT at room temperature under gentle stirring and washed out by R R R R20 R30 R30 with PBT. After R with staining buffer, the embryos were stained in 1-ml staining buffer with $4.5 \mu \mathrm{l} \mathrm{NBT}$ and $3 \mu \mathrm{l}$ BCIP added. Embryos were counterstained with DAPI and embedded in glycerol for bright field microscopy.

\section{Buffers}

The buffers used were: $10 \times$ PBS: $320 \mathrm{~g} \mathrm{NaCl}+8 \mathrm{~g} \mathrm{KCl}+$ 8 g KH2PO4 + 46 g Na2HPO4, add dH2O to $41, \mathrm{pH}=7$; PBT: PBS plus $0.02 \%$ Tween 20 ; stirred with $0.5 \mathrm{ml}$ DEPC per liter for $30 \mathrm{~min}$; autoclaved Hybe-A: $10 \mathrm{ml}$ deionized formamide, $5 \mathrm{ml}$ of $20 \times \mathrm{SSC}, \mathrm{pH} 5.5$ with $\mathrm{HCl}$, fill to $20 \mathrm{ml}$ with dest. water. Then add $0.4 \mathrm{ml}$ of $10 \mathrm{mg} / \mathrm{ml}$ of boiled sonicated salmon testis DNA, $100 \mu \mathrm{l}$ of $20 \mathrm{mg} / \mathrm{ml}$ tRNA, $20 \mu \mathrm{l}$ of $50 \mathrm{mg} / \mathrm{ml}$ heparin stock; Hybe-B: $50 \%$

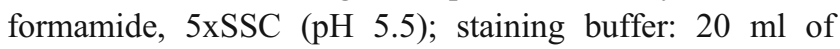
$0.1 \mathrm{M}$ Tris $\mathrm{pH} 9.5,1 \mathrm{ml}$ of $1 \mathrm{M} \mathrm{MgCl}_{2}, 400 \mu \mathrm{l}$ of $5 \mathrm{M}$ $\mathrm{NaCl}, 100 \mu \mathrm{l}$ of $20 \%$ Tween 20 .

\section{Results and discussion}

Rotifer culture and egg collection

Brachionus is pelagic in behavior and small in size (400$500-\mu \mathrm{m}$ body length) and shows sexual or parthenogenetic reproduction depending on environmental cues. The eggs are shed, attached to the posterior end of the mother, and carried around. One individual can carry up to four parthenogenetic eggs at a time under optimal culture conditions. B. plicatilis laboratory culture is simple and cheap and does not require much space (see "Material and methods" for details). It can be cultured in seawater in 1-1 bottles and fed on separately cultured marine phytoplankton. The culture can be maintained at room temperature. At $28^{\circ} \mathrm{C}$, the reproduction is highest with the culture doubling within 1 day. Males were not observed under these conditions suggesting a predominantly parthenogenetic mode of reproduction. Animals can be collected from the medium by filtration and harsh mechanical shocks separate the eggs from the parent animals. Together with the fast embryonic development $(24 \mathrm{~h})$, it is possible to obtain an almost unlimited amount of embryos all year round. Rotifer embryos are prone to osmotic implosion upon buffer changes. Although they resume their normal form afterwards, it is likely that some disruption of morphology occurs. Adding fixative or buffers in many small steps circumvents this problem.

\section{Permeabilization of the eggshell}

The eggshell of living and fixed embryos is rather impermeable even for small molecules and its chemical composition is not known in detail. We note that in a given batch there are always some embryos that are accessible to staining. We assume that the eggshell is damaged in these individuals by mechanical force during the manipulations. This happens more frequently to late-stage embryos while cleavage stages are almost never recovered this way. We therefore sought to find a method for shell permeabilization.

Living and freshly fixed embryos are not accessible to small fluorescent dyes (DAPI, Hoechst) unless they are incubated in methanol. However, this treatment does not enable the larger phalloidin to enter the embryo while adults in the same batch are staining strongly. We were not successful in identifying a chemical or enzyme which would digest the shell and render it permeable for larger molecules (see the "Materials and methods" section for the list of chemicals and enzymes tested). We found, however, that mechanical shearing is a means to raise the portion of eggs with ruptured eggshell (see "Materials and methods" for details). However, there is a tradeoff between opening or removing the eggshell and destruction of the embryos. As the embryonic material is virtually unlimited, the $10 \%$ yield of early-stage embryos that are rendered permeable by this treatment is high enough to analyze in situ patterns in all stages of embryogenesis within one batch (see for instance phalloidin staining in Fig. 2).

\section{Visualization of embryogenesis}

For the study of early development, a technique for visualizing cell outlines is useful. We used fluorescently labeled phalloidin as marker of the F-actin component of the cytoskeleton which resulted in a good visualization of cell outlines (Fig. 2). Our staining protocol will allow a precise description of gastrulation and organogenesis as a basis for the interpretation of expression patterns.

Whole-mount in situ hybridization of Pax-6

After successful removal of the eggshell, a standard WMISH protocol using proteinase $\mathrm{K}$ treatment was able to detect transcripts during embryogenesis. We cloned a 
fragment of Bp-Pax-6 and verified its orthology by sequence analysis (see alignment and topology of sequence similarities in S1 and S2, respectively). Whole-mount in situ hybridization with a Pax-6 antisense RNA probe is shown in Fig. 3. Using a counterstaining with DAPI, embryos are staged according to their number of cells. Expression of Bp-Pax-6 sets in at a stage which is, judging from the number of cells, probably near the onset of organogenesis. Expression is more or less bilaterally symmetric and is initially situated as a broad, patchy, curved domain laterally in the anterior half of the embryo (Fig. 3a). Expression is patchy only in the beginning; at later stages, the patches fuse (Fig. 3b,c). Judging from the DAPI staining, each patch might correspond to an
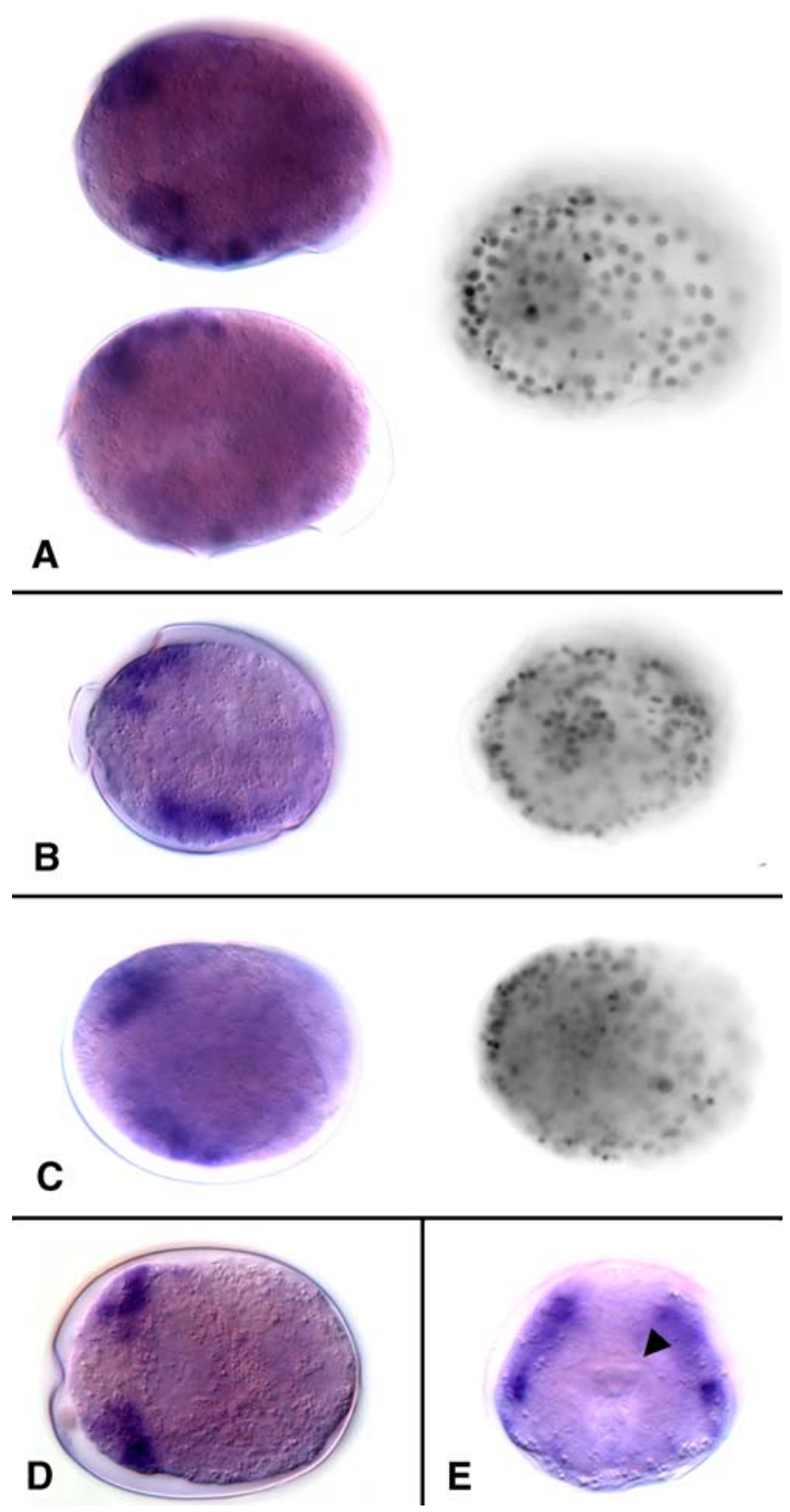

individual cell expressing the gene. Later on, the expression domain contracts (possibly a result of the decreasing size of embryonic cells) and moves anteriorly and dorsad. This process continues (Fig. 3b,c) until the final form of Bp-Pax6 expression in late development is reached (Fig. 3d,e). This expression persists until cellular differentiation is well underway (the rods of the mastax are visible-black arrowhead in Fig. 3e). Viewed from the dorsal perspective, the expression has now the form of a narrow transverse stripe situated almost at the anterior end on each body side. In the frontal view, it appears that the expression is broadly extended in the dorsoventral dimension (Fig. 3e).

The techniques presented here and the transparency of all stages of Brachionus development allows visualization of development and gene expression in the rotifer $B$. plicatilis. Recent EST projects (Suga et al. 2007; Witek et al. personal communication) and reports on transfection in this species (Shearer and Snell 2007) indicate that RNAi mediated gene knockdown may be feasible. The short generation time together with the high density of the rotifers in culture and the very restricted spatial requirement allows for keeping many stocks separately which is a prerequisite for transgenesis approaches. The production of storable resting eggs is a very attractive means to keep stock-keeping costs low. Moreover, its transparency allows visualization of development and gene expression in the rotifer Brachionus plicatilis. Together, these advances recommend $B$. plicatilis as lophotrochozoan model system for comparative analysis of gene expression.

Several interesting questions can now be addressed: Where are the genes involved in ecdysozoan and deuterostome metamerization expressed in this species representing a basal protostome branch? What does that tell us about the evolution of segmentation?

Another topic is the potential convergent evolution between the developmental mechanisms in $B$. plicatilis and the nematode Caenorhabditis elegans. Both organisms independently adapted their ontogeny to a very short life

Fig. 3 WMISH of Bp-Pax-6 in B. plicatilis embryos. Five experimental embryos are shown (a-e), ordered from top to down according to their developmental stage. The anterior end is oriented to the left except for panel e. DAPI counterstaining is shown for embryos a-c to the right. a The earliest stage in which expression of Bp-Pax-6 is observed. The two pictures to the left show different focal planes in order to show that the expression is symmetric. The expression domains are situated laterally and subdivided in patches which might correspond to single cells expressing Bp-Pax-6. b Expression becomes continuous and contracts towards anterior. $\mathbf{c}$ As cell number increases, the expression domains come to lie more anteriorly. d, e The latest stages scored for Bp-Pax- 6 expression. d Expression is now restricted to transverse lateral stripes in a subapical anterior position. e Frontal view, showing the dorsoventral extension of the late expression of Pax-6. The mastax ("jaw") is already visible at this stage (arrowhead). Note the damaged eggshells visible at the periphery of the embryos in $\mathbf{b}, \mathbf{c}$, and $\mathbf{d}$ 
span in combination with a small body size and fixed number of cells (eutely). Furthermore, the evolutionary origin of the corona (wheel organ) and the other peculiar autapomorphies of $B$. plicatilis and/or rotifers as a whole can now be studied using gene expression data as an additional source of information.

Acknowledgements The authors wish to acknowledge Wim Damen for degenerate primer sequences for the cloning of Bp-Pax-6, Ernst Wimmer for discussions and inspiring environment. and Drs. Boschetti and Ricci for sharing their observations on Brachionus embryology and culture.

Open Access This article is distributed under the terms of the Creative Commons Attribution Noncommercial License which permits any noncommercial use, distribution, and reproduction in any medium, provided the original author(s) and source are credited.

\section{References}

Boschetti C, Ricci C, Sotgia C, Fascio U (2005) The development of a bdelloid egg: a contribution after 100 years. Hydrobiologia 546:1-9

Castellano Paez ME, Kurokura H, Kasahara S (1988) Embryonic development of amictic eggs of a rotifer Brachionus plicatilis. J Facul Appl Biol Sci Hiroshima Univ 27:93-99

deBeauchamp P (1956) Le developpement de Ploesoma hudsoni (Imhof) et l'origine des feuillets chez les rotiferes. Bulletin de la Societé Zoologique de France 81:374-383

Dunn CW, Hejnol A, Matus DQ, Pang K, Browne WE, Smith SA, Seaver E, Rouse GW, Obst M, Edgecombe GD, Sorensen MV, Haddock SH, Schmidt-Rhaesa A, Okusu A, Kristensen RM, Wheeler WC, Martindale MQ, Giribet G (2008) Broad phyloge- nomic sampling improves resolution of the animal tree of life. Nature 452:745-749

Gomez A, Serra M, Carvalho GR, Lunt DH (2002) Speciation in ancient cryptic species complexes: evidence from the molecular phylogeny of Brachionus plicatilis (Rotifera). Evolution 56: $1431-1444$

Jennings HS (1896) The early development of Asplancha herrickii (de Guerne). Bull Mus Comp Zool., Harvard College 30:1-117

Lechner M (1966) Untersuchungen zur Embryonalentwicklung des Rädertieres Asplancha girodie de Guerne. Roux' Archiv für Entwicklungsmechanik 157:117-173

Nachtwey R (1925) Untersuchungen über die Keimbahn, Organogenese und Anatomie von Asplancha priodonta (Gosse). Zeitschrift für wissenschaftliche Zoologie 126:439-492

Pray F (1965) Studies on the early development of the rotifer Monostyla cornuta (Müller). Trans Am Microsc Soc 84:210-216

Shearer TL, Snell TW (2007) Transfection of siRNA into Brachionus plicatilis (Rotifera). Hydrobiologia 593:141-145

Suatoni E, Vicario S, Rice S, Snell T, Caccone A (2006) An analysis of species boundaries and biogeographic patterns in a cryptic species complex: the rotifer-Brachionus plicatilis. Mol Phylogenet Evol 41:86-98

Suga K, Mark Welch D, Tanaka Y, Sakakura Y, Hagiwara A (2007) Analysis of expressed sequence tags of the cyclically parthenogenetic rotifer Brachionus plicatilis. PLoS ONE 2:e671

Tamura K, Dudley J, Nei M, Kumar S (2007) MEGA4: Molecular Evolutionary Genetics Analysis (MEGA) software version 4.0. Mol Biol Evol 24:1596-1599

Tannreuther GW (1920) The development of Asplancha ebbesbornii (Rotifer). J Morphol 33:389-437

Tessin G (1886) Über die Eibildung und Entwicklung der Rotatorien. Zeitschrift für wissenschaftliche Zoologie 44:273-302

Welch DM (2001) Early contributions of molecular phylogenetics to understanding the evolution of Rotifera. Hydrobiologia 446447:315-322

Zelinka (1891) Studien über Räderthiere III. Zur Entwicklungsgeschichte der Räderthiere nebst Bemerkungen über ihre Anatomie und Biologie. Zeitschrift für wissenschaftliche Zoologie 53:1-159 